\title{
Commutativity of Linear Time-Varying Differential Systems with Nonzero Initial Conditions: A Review and Some New Extensions
}

\author{
Muhammet Koksal' ${ }^{1}$ and Mehmet Emir Koksal ${ }^{2}$ \\ ${ }^{1}$ Computer Engineering Department, Halic University, Siracevizler Street, no. 29, 34363 Sisli, \\ Istanbul, Turkey \\ ${ }^{2}$ Department of Elementary Mathematics Education, Mevlana University, 42003 Konya, Turkey
}

Correspondence should be addressed to Muhammet Koksal, muhammetkoksal@halic.edu.tr

Received 18 January 2011; Revised 21 June 2011; Accepted 12 July 2011

Academic Editor: P. Liatsis

Copyright (C) 2011 M. Koksal and M. E. Koksal. This is an open access article distributed under the Creative Commons Attribution License, which permits unrestricted use, distribution, and reproduction in any medium, provided the original work is properly cited.

\begin{abstract}
Necessary and sufficient conditions for the commutativity of linear time-varying systems are derived in the case of nonzero initial conditions. It is shown that some commutative class of linear time-varying systems may not commute with arbitrary initial conditions. In this respect, commutativity of Euler differential systems is investigated. Explicit commutativity conditions for the fifth-order systems are solved. New results about the effects of commutativity on system sensitivity and disturbance properties are presented, which is very important for network design and industrial applications where many of the systems are composed of subsystems cooperating one after another in a chain. The results are supported by examples treated either analytically or numerically.
\end{abstract}

\section{Introduction}

When two linear time-varying single-input single-output dynamical systems A and B are connected in cascade (or series), the input-output relation of the combined system depends on the parameters of both systems and on which appears first. If both of the connections $A B$ and BA have the same input-output pairs irrespective of the applied input, then we say that these systems are commutative systems; in this case $\mathrm{AB}$ and $\mathrm{BA}$ are equivalent, that is, $\mathrm{AB}=$ BA.

Most of the industrial processes and network designs contain subsystems or subnetworks which are arranged in a cascaded fashion or in a chain, which can be called a serial composition as well. If the change of the order of any two subunits in this chain does 
not affect the input-output relation of the whole system while it is creating some advantages on the sensitivity, stability, disturbance or noise, or other similar properties, the use of the optimum sequence is inevitable. Therefore, the study of the commutativity conditions is very important from theoretical and as well as practical point of views. And, this paper is aimed at closing the gap in the theory on the related topic.

An exhaustive study of the commutativity of the linear time-varying dynamical systems which can be referred as differential systems, due to their mathematical representations given in Section 2, was introduced in 1988 by the author [1]. This work has been the basic reference for years since it includes the most general necessary and sufficient commutativity conditions for systems of any order but without initial conditions. All the previous results concerning the commutativity conditions were shown to be deduced from the main theorem in that paper. Namely, those for the first-order [2], second-order [3-5], third-order [6], and fourth-order $[7,8]$ systems.

In addition to the deductions of the commutativity conditions for low-order systems, the main reference [1] covers some general results in the form of corollaries or theorems of minor importance. For example, Corollary 3.2 states that for the commutativity of two linear systems it is required that either both systems are time-invariant or both systems are time-varying, which is a result originally stated by Marshall [2]. Corollary 3.3 states the commutativity of identical time-varying systems with arbitrary time-invariant forward and feedback path gains, which is originally proved by the author himself for secondorder systems [5]. Theorem 5.1 deals with the unconditional commutativity property of Euler Systems in the light of the stated general necessary and sufficient conditions, which is originally treated in the undistributed report [7] and presented in a national conference [8].

Although the exhaustive study [1] included all the previous results reported until its publication, it was not claimed to be the completion of the research on the subject. On the contrary, it forecasted open questions on the commutativity conditions for systems with initial conditions and on the role of the system performance characteristics such as noise, sensitivity, and disturbance, in the cascade connected control or communication networks. Later, some new results on these subjects have appeared in the national [9] and international $[10,11]$ conference proceedings.

The commutativity conditions with nonzero initial conditions were derived in $[9,10]$. It was shown that two initially relaxed commutative systems may not be commutative when they have nonzero initial conditions; further, commutativity with arbitrary initial conditions requires additional constraints on the coefficients of the differential equations describing the systems. The importance of commutativity as far as the system sensitivity is concerned was reported in $[9,11]$. It was shown that for the same input-output relation, one system could be much less sensitive to system parameters than the other if the subsystems are commuted.

Although the above results are important from both theoretical and application point of views, it is believed that they have not been widely and sufficiently announced at least in an international journal paper. This is due to time limitations of the presentations in the conferences and page limitations in their proceedings which always sound less than journals. Further, some errors in the mentioned literature of major importance but minor reflection need to be corrected. Therefore, the scope of this paper is confined mainly on the explicit commutativity conditions for linear time-varying differential systems with nonzero initial conditions and the effects of commutativity on the system sensitivity. Further, the analytic solution of explicit commutativity conditions for the fifth-order systems is introduced in the literature for the first time. Another original point is the derivation of explicit commutativity 
conditions for Euler systems which are always commutative without initial conditions. Commutativity of time-invariant linear systems with nonzero initial conditions is also studied as another particular case. Further, new results are obtained for the commutativity of feedback systems with nonzero initial conditions.

This paper, as a survey or a tutorial on the commutativity with nonzero initial conditions, is mainly aimed at presenting the above-mentioned original items of theoretical values and moreover to highlight the importance of commutativity on industrial applications or engineering designs where most of the subsystems are connected in cascade or in a chain.

The rest of the paper is organized as follows: in the following section, the problem of commutativity is defined and for the sake of completeness the main results previously obtained in [1] are summarized as Theorem 2.1 without proof. In Section 3, the commutativity conditions for systems with initial conditions are introduced by Theorem 3.1; some results of minor importance in the above-mentioned literature are correctly stated. Particular results including feedback systems are presented. Section 4 includes the explicit solution of the commutativity conditions for the fifth-order systems. Examples showing the roles of commutativity on system disturbance and system sensitivity to parameter values are presented in Section 6 to show the practical importance of the results. Finally the last section is devoted to conclusions.

\section{First Commutativity Conditions}

Let the two linear time-varying dynamical systems $\mathrm{A}$ and $\mathrm{B}$ be represented by $n$th order linear differential equations with variable coefficients $a_{i}(t), b_{i}(t), i=1,2, \ldots, n$, respectively, as follows:

$$
\begin{aligned}
& \text { A: } \quad \sum_{i=0}^{n} a_{i}(t) \frac{d^{i}}{d t^{i}} y_{1}(t)=x_{1}(t), \quad a_{n}(t) \neq 0, \\
& \text { B: } \sum_{i=0}^{n} b_{i}(t) \frac{d^{i}}{d t^{i}} y_{2}(t)=x_{2}(t)
\end{aligned}
$$

where $a_{i}(\cdot)$ and $b_{i}(\cdot)$ are piecewise continuous mappings from $R$ into $R$ which is the real line; $x_{i}$ and $y_{i}$ are the input and output of the systems $\mathrm{A}(i=1)$ and $\mathrm{B}(i=2)$. It is assumed that $x_{i}(\cdot): R \rightarrow R$ is also piecewise continuous. Although $a_{n}(t) \neq 0$, that is, $\mathrm{A}$ is always assumed an $n$th order dynamical system, $(n \geq 1)$ it is allowed that $b_{i}(t)=0$ for $i=n, n-$ $1, \ldots, m \geq 0$, whilst $b_{m}(t) \neq 0$, which means B is an $m$ th order system; $m$ is assumed to be equal to $n$ unless otherwise stated. It is well known [12] that both systems and hence their interconnections described in the sequel have unique solutions under the cited conditions about the coefficients $a_{i}(t)$ and $b_{i}(t)$ unless $a_{n}\left(t_{0}\right) \neq 0$ and $b_{m}\left(t_{0}\right) \neq 0$, where $t_{0}$ is the initial time, which is a fact that will not be emphasized in this paper anymore.

For the cascade connection AB shown in Figure 1(a), the output $y_{1}(t)$ of $\mathrm{A}$ is equal to the input $x_{2}(t)$ of $\mathrm{B}$; so that letting $y_{1}(t)=x_{2}(t)$ and replacing $(2.1 \mathrm{~b})$ into $(2.1 \mathrm{a})$, the following differential equation is obtained

$$
\sum_{k=0}^{n} a_{k}(t) \sum_{i=0}^{m} \frac{d^{k}}{d t^{k}}\left[b_{i}(t) y^{(i)}(t)\right]=x(t)
$$




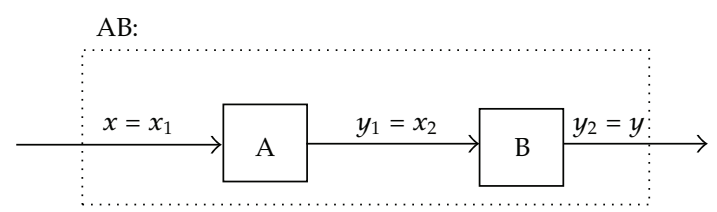

(a)

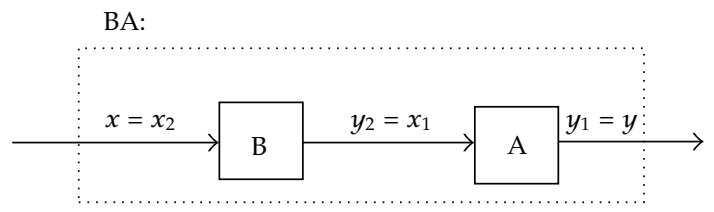

(b)

Figure 1: Cascade connection of the differential systems described by (2.1a) and (2.1b).

where $x(t)=x_{1}(t)$ and $y(t)=y_{2}(t)$ are the input and output of the cascaded system AB, respectively; the superscript in the parenthesis denotes $i$ th derivative. Note that $B$ is assumed of degree $m \leq n$. Performing the $k$ th derivative on the product $b_{i}(t) y^{i}(t)$ and rearranging, (2.2) reduces to the following $(n+m)$ th order differential equation describing $\mathrm{AB}$ :

$$
\sum_{r=0}^{n+m}\left[\sum_{i=\max (0, r-m)}^{n} \sum_{k=\max (0, i-r)}^{\min (i, i+m-r)} \frac{i !}{k !(i-k) !} a_{i} b_{r+k-i}^{(k)}\right] y^{(r)}=x
$$

For the connection BA shown in Figure 1(b), the input $x(t)=x_{2}(t), y_{2}(t)=x_{1}(t)$, the output $y(t)=y_{1}(t)$, and a similar equation to (2.3a) are obtained with the replacements of $n$, $m, a, b$ by $m, n, b, a$, respectively. The result is

$$
\sum_{r=0}^{m+n}\left[\sum_{i=\max (0, r-n)}^{m} \sum_{k=\max (0, i-r)}^{\min (i, i+n-r)} \frac{i !}{k !(i-k) !} b_{i} a_{r+k-i}^{(k)}\right] y^{(r)}=x .
$$

When the two differential equations in (2.3a), (2.3b) are identical except at a finite number of points in every finite subinterval of $R$ (these may be the discontinuity points of the coefficients $a_{i}(t)$ and $b_{i}(t)$ and their derivatives), the connections $\mathrm{AB}$ and $\mathrm{BA}$ will have the same output $y$ for the same input $x$ if the initial conditions for subsystems A and B are zero. When the coefficients of the derivatives of the same orders are equated, the necessary and sufficient conditions expressed by the following theorem can be obtained [1].

Theorem 2.1 (Koksal 1). The necessary and sufficient conditions for a linear time-varying system of order $n$ (System A) to be commutative with another linear time-varying system of the same or lower 
$\operatorname{order}($ System B), in the case of zero initial conditions, are that

(i) the coefficients of the differential equation describing the second system must be expressed in terms of the coefficients of the differential equation describing the first system through $n+1$ equations

$$
b_{i}=\sum_{j=n}^{i} c_{i} f_{i, j}, \quad i=n, n-1, \ldots, 0
$$

involving $(n+1)$ properly chosen constants $c_{n}, c_{n-1}, \ldots, c_{0}$, where

$$
\begin{gathered}
f_{i, j}=f_{i, j}\left(a_{n}, a_{n-1}, \ldots, a_{n+i-j}\right), \\
f_{i, j}=a_{i}, \text { for } j=n, i=n, n-1, \ldots, 0, \\
f_{i, j}=a_{i}^{i / n}, \quad \text { for } j=i=n-1, n-2, \ldots, 0, n \geq 1, \\
f_{i, j}=\frac{1}{n} a_{n}^{i / n} \int a_{n}^{-(i+n) / n}\left[\sum_{k=i+1}^{j} \sum_{s=\max (1, k-n+2)}^{\min (k, k-i+1)} \frac{k !}{s !(k-s) !} a_{n+i+s-k-1}^{(s)} f_{k, j}\right. \\
\left.-\sum_{k=\max (i+1, n+i-j)}^{n} \sum_{s=\max (1, k-n+2)}^{k+(k-s) !} a_{k}^{(s)} f_{n+i+s-k-1, j}^{(s)}\right] d t \\
\text { for } j=n-1, n-2, \ldots, 1, \quad i=j-1, j-2, \ldots, 0, \quad n \geq 2 ;
\end{gathered}
$$

(ii) further, with the same constants $c_{i}$ 's, the coefficients of the first system must satisfy $(n-1)$ nonlinear algebraic equations

$$
\sum_{j=n-1}^{1} F_{i, j} c_{j}=0, \quad \text { for } i=n-1, n-2, \ldots, 1
$$

which involve the derivatives of the coefficients as well due to the expression

$$
\begin{aligned}
F_{i, j}= & \sum_{k=\max (1, i-j)}^{n} \sum_{s=\max (1, k-i+1)}^{\min (k, k-i+j+1)} \frac{k !}{s !(k-s) !} a_{k} f_{i+s-k-1, j}^{(s)} \\
& -\sum_{k=1}^{j} \sum_{s=\max (1, k-i+1)}^{k} \frac{k !}{s !(k-s) !} f_{k, j} a_{i+s-k-1}^{(s)} \quad \text { for } i, j=n-1, n-2, \ldots, 0 .
\end{aligned}
$$

Although this theorem is apparently stated and originally proved for $n=m$, that is, for the commutativity of equal order systems, it should be remarked that it can easily be reduced for the case $m<n$ by simply choosing $c_{n}, c_{n-1}, \ldots, c_{m+1}=0$, which results with 
$b_{n}=b_{n-1}=\cdots=b_{m+1}=0$. Hence, it is valid for systems of any order. Another remark to be noted is that a decreasing order indexing is used for convenience in all of the subscripts and summations in (2.4a) and (2.5a) in accordance with the order of computations from simple to complex; this order corresponds to the decreasing order of derivatives and coefficients in the original equations (2.1a), (2.1b).

The above theorem expresses the necessary and sufficient conditions for systems A and $B$ in the case of zero initial conditions for the differential systems in (2.1a), (2.1b). Although the initial clue for the proof is given above, the full proof is exhaustive and given in [1] which also discusses the consequences of the theorem. Therefore, it is not included in this paper. Instead, the case of nonzero initial conditions is considered next.

\section{Second Commutativity Conditions}

Let the systems in (2.1a), (2.1b) be commutative when they are initially relaxed, that is, they satisfy the conditions of Theorem 2.1. Consider now the nonzero initial conditions expressed by

$$
\begin{aligned}
& Y_{A}=\left[\begin{array}{llll}
y_{1}^{(0)} & y_{1}^{(1)} \cdots y_{1}^{(m-1)} \vdots y_{1}^{(m)} \quad y_{1}^{(m-1)} \cdots y_{1}^{(n-1)}
\end{array}\right]^{T}=\left[\begin{array}{l}
Y_{A 1} \\
Y_{A 2}
\end{array}\right], \\
& Y_{B}=\left[\begin{array}{llll}
y_{2}^{(0)} & y_{2}^{(1)} \cdots & y_{2}^{(m-1)}
\end{array}\right]^{T} \text {, }
\end{aligned}
$$

where $Y_{A 1}$ and $Y_{A 2}$ express the vector of the first $m$ and the remaining $n-m$ initial values of $y_{1}(t)$ and it is derivatives according to the partitioning in (3.1a). With these initial conditions, the commutativity requirements are expressed by the following theorem.

Theorem 3.1 (Koksal 2). The necessary and sufficient conditions for the commutativity of two linear time-varying systems $A$ and $B$ which are described by the differential equations in (2.1a) and (2.1b), and with the initial conditions in (3.1a) and (3.1b), respectively, are

(i) the conditions of Theorem 2.1 on the coefficients (2.4a), (2.5a) be satisfied,

(ii) the initial conditions satisfy the following at the initial time $t_{0}$ :

$$
\stackrel{(n)}{(m)}\left[\begin{array}{c}
Y_{A} \\
A_{2}^{-1}\left(Y_{B}-A_{1} Y_{A}\right)
\end{array}\right]=\left[\begin{array}{c}
Y_{B} \\
B_{2}^{-1}\left(Y_{A}-B_{1} Y_{B}\right)
\end{array}\right] \begin{aligned}
& (m) \\
& (n)
\end{aligned}
$$

or equivalently,

$$
\begin{aligned}
& (n) \quad(m) \quad(n) \quad(m) \\
& \left\{\begin{array}{c}
(n) \\
(m)
\end{array}\left[\begin{array}{cc}
I & 0 \\
-A_{2}^{-1} A_{1} & A_{2}^{-1}
\end{array}\right]-\begin{array}{c}
(m) \\
(n)
\end{array}\left[\begin{array}{cc}
0 & I \\
B_{2}^{-1} & -B_{2}^{-1} B_{1}
\end{array}\right]\right\}\left[\begin{array}{l}
Y_{A} \\
Y_{B}
\end{array}\right]=[0],
\end{aligned}
$$

where the matrices $A_{1}, A_{2}, B_{1}, B_{2}$ are defined in the proof (see (3.8a)-(3.8d)). 
Proof. Having satisfied the conditions of Theorem 2.1, the differential equations in (2.3a) and (2.3b) will have the same coefficients. For the same input, they will have identical solutions if their initial conditions are also equal.

For the connection $\mathrm{AB}, y=y_{2}$, and for $\mathrm{BA}, y=y_{1}$; hence to have the same initial conditions for $y$ in (2.3a) and (2.3b),

$$
y^{(k)}=y_{1}^{(k)}=y_{2}^{k} ; \quad \text { for } k=0,1, \ldots, n+m-1
$$

must be satisfied at the initial time $t=t_{0}$. On the other hand, using $x_{2}=y_{1}$ for $\mathrm{AB}$ and $x_{1}=y_{2}$ for BA in (2.1b) and (2.1a),

$$
\begin{aligned}
& y_{1}=\sum_{j=0}^{m} b_{j} y_{2}^{(j)}, \\
& y_{2}=\sum_{j=0}^{n} a_{j} y_{1}^{(j)}
\end{aligned}
$$

are obtained for the connections $\mathrm{AB}$ and $\mathrm{BA}$, respectively. Using the formula

$$
\frac{d^{k}}{d t^{k}}(u v)=\sum_{s=0}^{k} \frac{k !}{s !(k-s) !} u^{(s)} v^{(k-s)}
$$

the $k$ th derivative of a product

$$
\begin{aligned}
& y_{1}^{(k)}=\sum_{j=0}^{m} \sum_{s=0}^{k} \frac{k !}{s !(k-s) !} b_{j}^{(s)} y_{2}^{(i+k-s)}, \quad k=0,1, \ldots, n-1, \\
& y_{2}^{(k)}=\sum_{j=0}^{n} \sum_{s=0}^{k} \frac{k !}{s !(k-s) !} a_{j}^{(s)} y_{1}^{(j+k-s)}, \quad k=0,1, \ldots, m-1
\end{aligned}
$$

can be obtained from (3.4a) and (3.4b), respectively. 
The last two equations can be written in matrix form as

$$
\begin{gathered}
B_{1} Y_{B}+B_{2}\left[\begin{array}{c}
y_{2}^{(m)} \\
y_{2}^{(m+1)} \\
\vdots \\
y_{2}^{(n+m-1)}
\end{array}\right]=Y_{A} \\
A_{1} Y_{A}+A_{2}\left[\begin{array}{c}
y_{1}^{(n)} \\
y_{1}^{(n+1)} \\
\vdots \\
y_{1}^{(n+m-1)}
\end{array}\right]=Y_{B}
\end{gathered}
$$

where the entries $a_{i j}^{\prime}\left(a_{i j}^{\prime \prime}, b_{i j}^{\prime}, b_{i j}^{\prime \prime}\right)$ of the coefficient matrix $A_{1}\left(A_{2}, B_{1}, B_{2}\right)$ are defined by

$$
\begin{aligned}
a_{i j}^{\prime} & =\sum_{s=\max (0, i-j)}^{i-1} \frac{(i-1) !}{s !(i-1-s) !} a_{j-i+s^{\prime}}^{(s)} \text { for } i=1,2, \ldots, m, j=1,2, \ldots, n, \\
a_{i j}^{\prime \prime} & =\sum_{s=0}^{i-j} \frac{(i-1) !}{s !(i-1-s) !} a_{j-i+m+s^{\prime}}^{(s)} \text { for } i=1,2, \ldots, m, j=1,2, \ldots, i, \\
& =0, \quad \text { for } i=1,2, \ldots, m-1, j=i+1, i+2, \ldots, m, \\
b_{i j}^{\prime} & =\sum_{s=\max (0, i-j)}^{i-1} \frac{(i-1) !}{s !(i-1-s) !} b_{j-i+s^{\prime}}^{(s)} \text { for } i=1,2, \ldots, n, j=1,2, \ldots, m, \\
b_{i j}^{\prime \prime} & =\sum_{s=\max (0, i-j-m)}^{i-j} \frac{(i-1) !}{s !(i-1-s) !} b_{j-i+m+s^{\prime}}^{(s)} \quad \text { for } i=1,2, \ldots, n, j=1,2, \ldots, i, \\
& =0, \quad \text { for } i=1,2, \ldots, n-1, j=i+1, i+2, \ldots, n .
\end{aligned}
$$

The above expressions for the entries of the coefficient matrices in (3.7a), (3.7b) imply that $A_{1}, A_{2}, B_{1}, B_{2}$ are matrices of orders $m \times n, m \times m, n \times m, n \times n$, respectively. Further, the square matrices $A_{2}$ and $B_{2}$ are lower triangular matrices with nonzero diagonal elements $a_{n}\left(t_{0}\right)$ and $b_{m}\left(t_{0}\right)$, respectively; therefore, they have well-defined inverses. Hence, $y_{1}^{(k)}$ for $k=n, n+1, \ldots, n+m-1$ can be computed from (3.7b), and $y_{2}^{(k)}$ for $k=m, m+1, \ldots, m+n-1$ can be computed from (3.7a). If these computed values are inserted in (3.3), this equation can be rearranged in matrix from as in (3.2a) or equivalently (3.2b).

The linear time varying systems $\mathrm{A}$ and $\mathrm{B}$ are commutative for all inputs if and only if (1) $\mathrm{AB}$ and $\mathrm{BA}$ are described by identical differential equations and (2) this differential equation has the same initial conditions for $\mathrm{AB}$ and $\mathrm{BA}$ at the initial time $t_{0}$. Hence, in addition to the conditions of Theorem 2.1, the conditions obtained in (3.2a), (3.2b) for the equivalent initial conditions of (2.3a) and (2.3b) constitute the necessary and sufficient conditions of 
the commutatitivity of the systems A and B. Equation (2.3b) must be satisfied by the initial conditions of $\mathrm{A}$ and $\mathrm{B}$. Hence this completes the proof.

This theorem is stated improperly in the present literature $[9,10]$, which formulated that the second condition above as the coefficient matrix in (3.2b) be identically zero, which is an impossible situation unless $m=0$ and system B is an identity.

We now consider the consequences of Theorem 3.1 in the form of corollaries. First consider the commutativity of A and B for all arbitrary initial conditions. This is a case which we call absolute commutativity. Even the first condition about the coefficients is satisfied, the commutativity for all arbitrary initial conditions is not possible if $m \geq 1$. This is obvious from the first block of $m$ equations in (3.2a). If $m=0,(3.2 \mathrm{a}),(3.2 \mathrm{~b})$ imply that $\left(I-B_{2}^{-1}\right) Y_{A}=0$; that is, $B_{2}=I$ for the commutativity of all initial conditions. Equation (3.8d) gives that $B_{2}=$ $\operatorname{diag}\left[\begin{array}{llll}b_{0} & b_{0} & \cdots & b_{0}\end{array}\right]$; hence $b_{0}=1$, which means that system $\mathrm{B}$ is an identity. This result can be expressed by the following corollary.

Corollary 3.2 (Absolute Commutativity). There are no time-varying dynamical systems which are absolutely commutative at any time $t_{0}$. The only absolutely commutative system of a time-varying system of order $n \geq 1$ is the identity. Note that the corollary holds for time-invariant systems as well.

When the case of the special values of the initial conditions is considered for A and B at a particular initial time $t_{0}$ (conditional commutativity), the first $m$ rows and the remaining $n$ rows of $(3.2 b)$ can be expressed as follows:

$$
\begin{aligned}
& Y_{B}=Y_{A 1}, \\
& \text { (m) }(n-m) \quad(m) \quad(n-m)
\end{aligned}
$$

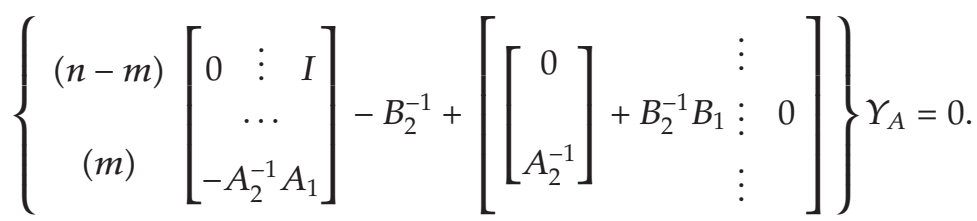

Corollary 3.3 (Conditional Commutativity). If the systems A and B of Theorem 3.1 are commutative with zero initial conditions, the necessary and sufficient conditions for their commutativity under nonzero initial conditions at time $t_{0}$ as well are

(i) the first m initial values of A expressed in (3.1a) must be identical with those of B expressed in (3.1b);

(ii) the initial condition vector of $A$, that is, $Y_{A}$ in (3.1a) must be in the null space of the value of the coefficient matrix in $(3.9 \mathrm{~b})$ at the initial time to $t_{0}$.

Corollary 3.4 (Conditional Commutativity of Systems of Equal Orders). If $A$ and $B$ are commutative systems with the same order $n$, for their commutativity with nonzero initial conditions at the initial time $t_{0}$, it is necessary and sufficient that both of these systems must have the same initial condition vectors $Y_{A}=Y_{B}$ in the null space of $n \times n$ matrix

$$
A_{2}^{-1}\left(I-A_{1}\right)-B_{2}^{-1}\left(I-B_{1}\right)
$$

at $t=t_{0}$.

This corollary follows from (3.9a), (3.9b) with $m=n$. 


\section{Reduction for Special Cases}

\subsection{Time-Invariant Systems with Nonzero Initial Conditions}

If the coefficients of linear systems in (2.1a) and (2.1b) are constant, A and B become timeinvariant. Since time-invariant linear systems with zero initial conditions are commutative, they commute [1]. Although primitive, this can be proved by considering the Laplace transform of (2.1a) and (2.1b) with zero initial conditions and showing that AB and BA have the same transfer function $H(s)$

$$
\frac{Y(s)}{X(s)}=H(s)=\frac{1}{\left(\sum_{i=1}^{n} a_{i} s^{i}\right)\left(\sum_{i=1}^{m} b_{i} s^{i}\right)},
$$

where $Y(s)$ and $X(s)$ are the Laplace Transforms of the input $x(t)$ and the output $y(t)$ of the connections $\mathrm{AB}$ and $\mathrm{BA}$. An alternate way would be to show that the conditions of Theorem 2.1 are satisfied. This necessitates the reduction of the formulas in (2.4a), (2.4b) and $(2.5 \mathrm{a}),(2.5 \mathrm{~b})$ for constant $a_{i}$ 's. Since the fact to be shown is obvious from the above discussion, this approach is omitted.

The commutativity of time-invariant systems with nonzero initial conditions, however is not straightforward. In this case, Corollaries 3.3 and 3.4 are valid for all initial times $t_{0}$, which is due to the time invariance of the coefficients. For the last reason, the expressions in (3.8a)-(3.8d) for the entries of the coefficient matrices in (3.2a), (3.2b), (3.7a), (3.7b), (3.9a), (3.9b) simplify to

$$
\begin{aligned}
& a_{i j}^{\prime}=a_{j-i}, \quad \text { for } i=1,2, \ldots, m, j=i, i+1, \ldots, n, \\
& a_{i j}^{\prime \prime}=a_{m-j-i}, \quad \text { for } i=1,2, \ldots, m, j=1,2, \ldots, i, \\
& b_{i j}^{\prime}=b_{j-i}, \quad \text { for } i=1,2, \ldots, n, j=i, i+1, \ldots, m, \\
& b_{i j}^{\prime \prime}=b_{m+j-i}, \quad \text { for } i=1,2, \ldots, n, j=\max (1, i-m), \ldots, i .
\end{aligned}
$$

All the other entries of $A_{1}, A_{2}, B_{1}, B_{2}$ which are not defined above are zero. Therefore, in addition to the lower triangular matrices $A_{2}$ and $B_{2}, A_{1}$ and $B_{1}$ become upper triangular (trapezoidal if $m<n$ ).

\subsection{Euler Systems with Nonzero Initial Conditions}

It is well known that linear time-varying systems described by Euler-type differential equations [13] are always commutative when the initial conditions are zero $[1,7,8]$. A detailed proof in [1] is given based on the impulse response representations of the individual systems as well as that of the interconnection. That paper also shows that the cascade connection of any two Euler systems is another Euler system, and further, all the commutative pairs of an Euler system are of the same type, that is, Euler-type. Although the proof can be done by showing that any Euler system A of order $n$ and another one B of the same or lower order satisfy the conditions of Theorem 2.1, the attempt here will be directly to the case of initial conditions. 
It is shown by a simple example in [10] that the commutativity of Euler systems with arbitrary nonzero initial conditions is not straightforward as in the case of time-invariant systems. Here in the following, the problem is treated on a general base as an application of the results obtained in the previous section.

The differential systems A and B described by (2.1a) and (2.1b), respectively, become Euler-type systems if the time-varying coefficients are set to

$$
\begin{aligned}
& a_{i}(t)=A_{i} t^{i}, \quad i=0,1, \ldots, n, \\
& b_{i}(t)=B_{i} t^{i}, \quad i=0,1, \ldots, m .
\end{aligned}
$$

Using the formula for the $s$ th derivative of the $k$ th power, that is,

$$
\frac{d^{s}}{d t^{s}} t^{k}= \begin{cases}\frac{k !}{(k-s) !} t^{k-s}, & \text { for } s=0,1, \ldots, k \\ 0, & \text { for } s>k\end{cases}
$$

all the previous results about the commutativity of Euler systems with nonzero initial conditions, in particular Theorem 3.1, Corollaries 3.3 and 3.4, can be derived with slight modifications. Since $a_{n}(0)=0, b_{m}(0)=0$ only for $t=0$, the initial time $t_{0}$ can be any real number except 0 . The mentioned modifications are on the expressions in (3.8a)-(3.8d) for the entries of the coefficient matrices $A_{1}, A_{2}, B_{1}, B_{2}$ which are used in the statement of the theorem and its mentioned corollaries. The Euler systems (3.8a)-(3.8d) reduce to the following:

$$
\begin{aligned}
& a_{i j}^{\prime}=t^{j-i} \sum_{s=0}^{i-1} \frac{(i-1) !}{s !(i-1-s) !} \frac{(j-i+s) !}{(j-i) !} A_{j-i+s}, \quad \text { for } i=1,2, \ldots, m, j=i, i+1, \ldots, n, \\
& a_{i j}^{\prime \prime}=t^{j-i+m} \sum_{s=0}^{i-j} \frac{(i-1) !}{s !(i-1-s) !} \frac{(j-i+m+s) !}{(j-i+m) !} A_{j-i+m+s}, \quad \text { for } i=1,2, \ldots, m, j=1,2, \ldots, i \\
& b_{i j}^{\prime}=t^{j-i} \sum_{s=0}^{i-1} \frac{(i-1) !}{s !(i-1-s) !} \frac{(j-i+s) !}{(j-i) !} B_{j-i+s}, \quad \text { for } i=1,2, \ldots, m, j=i, i+1, \ldots, m \\
& b_{i j}^{\prime \prime}=t^{j-i+m} \sum_{s=0}^{i-j} \frac{(i-1) !}{s !(i-1-s) !} \frac{(j-i+m+s) !}{(j-i+m) !} B_{j-i+m+s} \\
& \text { for } i=1,2, \ldots, n, \quad j=\max (1, i-m), \ldots, i
\end{aligned}
$$

Note that the entries $a_{i j}^{\prime}, a_{i j}^{\prime \prime}, b_{i j}^{\prime}, b_{i j}^{\prime \prime}$ of the matrices $A_{1}, A_{2}, B_{1}, B_{2}$ of orders $m \times n, m \times m, n \times n$, $n \times m$, which are not defined in $(4.5 \mathrm{a}),(4.5 \mathrm{~b}),(4.5 \mathrm{c}),(4.5 \mathrm{~d})$, respectively, are all zero. Hence as in the time-invariant case, $A_{1}$ and $B_{1}$ are upper triangular.

Examples to explicit forms of the coefficient matrices used to express the constraints on the initial conditions for commutativity will be given in Section 6. 
A:

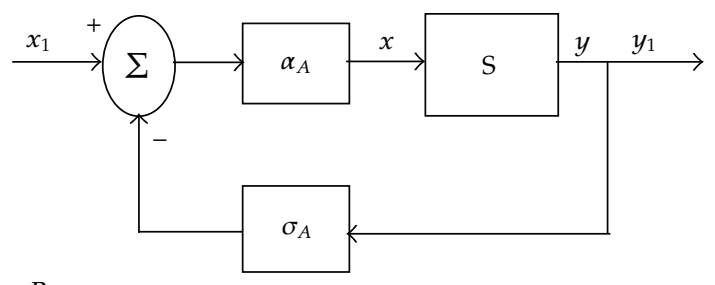

B:

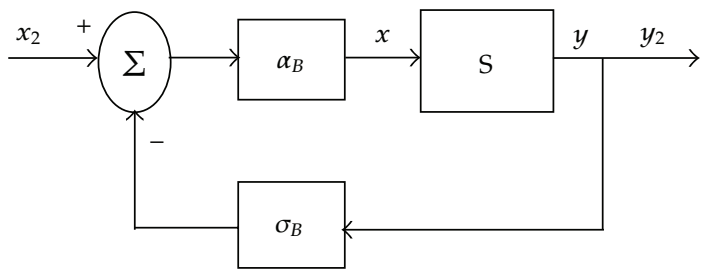

Figure 2: Feedback systems obtained from $S$ by constant forward and feedback path gains.

\subsection{Feedback Systems}

Due to their importance in automatic control theory, commutativity of feedback systems is expressed by a corollary.

Corollary 4.1. Any two versions $A$ and $B$ of a linear time-varying system $S$ simply obtained by constant forward and feedback path gains $\boldsymbol{\alpha}_{\mathbf{A}}$ and $\boldsymbol{\sigma}_{\mathrm{A}}, \boldsymbol{\alpha}_{\mathbf{B}}$ and $\boldsymbol{\sigma}_{\mathrm{B}}$, respectively, are commutative with any set of initial conditions of $S$ if and only if

$$
\alpha_{\mathrm{A}}\left(\sigma_{\mathrm{A}}-1\right)=\alpha_{\mathrm{B}}\left(\sigma_{\mathrm{B}}-1\right)
$$

where $\boldsymbol{\alpha}_{\mathrm{A}}, \boldsymbol{\alpha}_{\mathrm{B}} \neq \mathbf{0}$

Proof. Let the system $\mathrm{S}$ be described by

$$
\text { S: } \quad s_{n} \frac{d^{n}}{d t^{n}} y+s_{n-1} \frac{d^{n-1}}{d t^{n-1}} y+\cdots+s_{1} \frac{d}{d t} y+s_{0} y=x
$$

Then the feedback systems A and B shown in Figure 2 are described by (2.1a) and (2.1b), respectively, with

$$
\begin{aligned}
a_{i}=\frac{s_{i}}{\alpha_{A}} \quad \text { for } i=n, n-1, \ldots, 1, & a_{0}=\frac{s_{0}}{\alpha_{A}}+\sigma_{A}, \\
b_{i}=\frac{s_{i}}{\alpha_{B}} \quad \text { for } i=n, n-1, \ldots, 1, & b_{0}=\frac{s_{0}}{\alpha_{B}}+\sigma_{B} .
\end{aligned}
$$


Since these coefficients can be related as in (2.4a) with

$$
\begin{gathered}
c_{n}=\frac{\alpha_{A}}{\alpha_{B}}, \quad c_{0}=\sigma_{B}-\frac{\alpha_{A}}{\alpha_{B}} \sigma_{A}, \\
c_{i}=0, \quad \text { for } i=n-1, n-2, \ldots, 1
\end{gathered}
$$

and (2.5a) is satisfied due to (4.9b), conditions of Theorem 2.1 are satisfied. Hence, A and B are always commutative with zero initial conditions.

For the case of nonzero initial conditions, first note that $y_{1}=y_{2}=y$ so that A and B have the same initial conditions as $S$; so Corollary 3.4 is satisfied with $Y_{A}=Y_{B}=Y_{S}$. Then express $b_{i}$ 's in terms of $a_{i}{ }^{\prime}$ s by using (4.8) and (4.9a), (4.9b) as follows:

$$
\begin{gathered}
b_{i}=c_{n} a_{i}=\frac{\alpha_{A}}{\alpha_{B}} a_{i}, \quad i=n, n-1, \ldots, 1, \\
b_{0}=c_{n} a_{0}+c_{0}=\frac{\alpha_{A}}{\alpha_{B}} a_{0}+\left(\sigma_{B}-\frac{\alpha_{A}}{\alpha_{B}} \sigma_{A}\right) .
\end{gathered}
$$

Using these expressions in (3.8c), (3.8d) to evaluate the coefficient matrices $B_{1}, B_{2}$ appearing in (3.10), the following results are obtained:

$$
B_{1}=\frac{\alpha_{A}}{\alpha_{B}} A_{1}+c_{0} I, \quad B_{2}=\frac{\alpha_{A}}{\alpha_{B}} A_{2}
$$

Finally inserting these expressions in (3.10),

$$
\left[1-\sigma_{A}+\frac{\alpha_{B}}{\alpha_{A}}\left(\sigma_{B}-1\right)\right] A_{2}^{-1}
$$

is obtained. Since $A_{2}$ is nonsingular and $\alpha_{A} \neq 0, \alpha_{B} \neq 0$, the common initial condition vector can be arbitrary chosen if the scalar coefficient of $A_{2}^{-1}$ in (4.12) is zero; that is,

$$
\alpha_{A}\left(\sigma_{A}-1\right)=\alpha_{B}\left(\sigma_{B}-1\right) .
$$

Otherwise, A and B cannot commute with any nonzero initial conditions; hence this completes the proof.

\section{Explicit Formulas for the Fifth-Order Systems}

The explicit commutativity conditions are important from two points of views. The first is the ease for the applicability of the use of the commutativity conditions stated in Theorems 2.1 and 3.1. The second is their contribution on the development of the commutativity theory. In fact, the starting point of the commutativity theory considered in the sense of this paper is the explicit commutativity conditions suggested by Marshall for the first-order systems [2]. Later the explicit commutativity conditions for second-order systems [3-5] and those for third-order systems [6] constituted the base of the general commutativity conditions 
originally presented in [6]. Later the explicit conditions of commutativity for forth-order systems appeared in the undistributed work [7] and presented in [8]. All of these results were summarized in the main reference [1].

Here as in the following theorem, the explicit commutativity conditions for fifth-order linear time-varying systems are presented for the first time.

Theorem 5.1 (Koksal 3). The necessary and sufficient conditions for a fifth-order linear time-varying system of order $n$ (System A) to be commutative with another linear time-varying system of the fifthor lower-order (System B), in the case of zero initial conditions, are that

(i) the coefficients of the differential equation describing the second system must be expressed in terms of the coefficients of the differential equation describing the first system through 6 equations

$$
\left[\begin{array}{l}
b_{5} \\
b_{4} \\
b_{3} \\
b_{2} \\
b_{1} \\
b_{0}
\end{array}\right]=\left[\begin{array}{cccccc}
a_{5} & 0 & 0 & 0 & 0 & 0 \\
a_{4} & a_{5}^{4 / 5} & 0 & 0 & 0 & 0 \\
a_{3} & f_{34} & a_{5}^{3 / 5} & 0 & 0 & 0 \\
a_{2} & f_{24} & f_{23} & a_{5}^{2 / 5} & 0 & 0 \\
a_{1} & f_{14} & f_{13} & f_{12} & a_{5}^{1 / 5} & 0 \\
a_{0} & f_{04} & f_{03} & f_{02} & f_{01} & 1
\end{array}\right]\left[\begin{array}{c}
c_{5} \\
c_{4} \\
c_{3} \\
c_{2} \\
c_{1} \\
c_{0}
\end{array}\right]
$$

involving 6 properly chosen constants $c_{5}, c_{4}, c_{3}, c_{2}, c_{1}, c_{0}$, where

$$
\begin{aligned}
f_{34}= & \frac{2}{5} a_{5}^{-1 / 5}\left(2 a_{4}-\dot{a}_{5}\right), \quad f_{12}=\frac{1}{5} a_{5}^{-3 / 5}\left(2 a_{4}-3 \dot{a}_{5}\right), \\
f_{23}= & \frac{3}{5} a_{5}^{-2 / 5}\left(a_{4}-\dot{a}_{5}\right), \quad f_{01}=\frac{1}{5} a_{5}^{-4 / 5}\left(a_{4}-2 \dot{a}_{5}\right), \\
f_{24}= & \frac{2}{5} a_{5}^{-1 / 5}\left(2 a_{3}-\dot{a}_{4}\right)+\frac{1}{25} a_{5}^{-6 / 5}\left(-2 a_{4}^{2}+4 a_{4} \dot{a}_{5}+3\left(\dot{a}_{5}\right)^{2}\right), \\
f_{13}= & \frac{1}{5} a_{5}^{-2 / 5}\left(3 a_{3}-3 \dot{a}_{4}-\ddot{a}_{5}\right)+\frac{3}{25} a_{5}^{-7 / 5}\left(-a_{4}^{2}+3 a_{4} \dot{a}_{5}\right), \\
f_{02}= & \frac{1}{5} a_{5}^{-3 / 5}\left(2 a_{3}-3 \dot{a}_{4}+2 \ddot{a}_{5}\right)+\frac{3}{25} a_{5}^{-8 / 5}\left(-a_{4}^{2}+4 a_{4} \dot{a}_{5}-2\left(\dot{a}_{5}\right)^{2}\right), \\
f_{14}= & \frac{1}{5} a_{5}^{-1 / 5}\left(4 a_{2}-2 \dot{a}_{3}+\dddot{a}_{5}\right)+\frac{2}{25} a_{5}^{-6 / 5}\left(-2 a_{3} a_{4}+3 a_{3} \dot{a}_{5}+2 a_{4} \dot{a}_{4}+\dot{a}_{4} \dot{a}_{5}-6 \dot{a}_{5} \ddot{a}_{5}\right) \\
& +\frac{1}{125} a_{5}^{-11 / 25}\left(4 a_{4}^{3}-18 a_{4}^{2} \dot{a}_{5}-4 \dot{a}_{4} a_{5}^{2}+33\left(\dot{a}_{5}\right)^{3}\right), \\
= & \frac{1}{5} a_{5}^{-2 / 5}\left(3 a_{2}-3 \dot{a}_{3}+\ddot{a}_{4}+\dddot{a}_{5}\right)+\frac{7}{25} a_{5}^{-12 / 5}\left(a_{4}^{3}-6 a_{4}^{2} \dot{a}_{5}+5 a_{4}\left(\dot{a}_{5}\right)^{2}+6\left(\dot{a}_{5}\right)^{3}\right) \\
& +\frac{1}{125} a_{5}^{-7 / 25}\left(-6 a_{3} a_{4}+12 a_{3} \dot{a}_{5}+9 a_{4} \dot{a}_{4}-4 a_{4} \ddot{a}_{5}-7 \dot{a}_{4} \dot{a}_{5}-14 \dot{a}_{5} \ddot{a}_{5}\right),
\end{aligned}
$$




$$
\begin{aligned}
f_{04}= & \frac{1}{25} a_{5}^{-1 / 5}\left(20 a_{1}-10 \dot{a}_{2}+5 \dddot{a}_{4}-4 \ddot{a}_{5}\right) \\
& +\frac{1}{125} a_{5}^{-6 / 5}\left(-20 a_{2} a_{4}+40 a_{2} \dot{a}_{5}-10 a_{3}^{2}+30 a_{3} \dot{a}_{4}+20 \dot{a}_{3} a_{4}-10 a_{3} \ddot{a}_{5}-10 \dot{a}_{3} \dot{a}_{5}\right. \\
& \left.+40 a_{4} \ddot{a}_{4}-20 a_{4} \dddot{a}_{5}+35\left(\dot{a}_{4}\right)^{2}-55 \dot{a}_{4} \ddot{a}_{5}-45 \ddot{a}_{4} \dot{a}_{5}+46 \dot{a}_{5} \ddot{a}_{5}+42\left(\ddot{a}_{5}\right)^{2}\right) \\
& +\frac{1}{625} a_{5}^{-11 / 5}\left(60 a_{3} a_{4}^{2}-240 a_{3} a_{4} \dot{a}_{5}+90 a_{3}\left(\dot{a}_{5}\right)^{2}-90 a_{4}^{2} \dot{a}_{4}+20 a_{4}^{2} \ddot{a}_{5}\right. \\
& \left.\quad-140 a_{4} \dot{a}_{4} \dot{a}_{5}+440 a_{4} \dot{a}_{5} \ddot{a}_{5}+385 \dot{a}_{4}\left(\dot{a}_{5}\right)^{2}-594\left(\dot{a}_{5}\right)^{2} \ddot{a}_{5}\right) \\
& +\frac{11}{3125} a_{5}^{-16 / 5}\left(-5 a_{4}^{4}+40 a_{4}^{3} \dot{a}_{5}-40 a_{4}^{2}\left(\dot{a}_{5}\right)^{2}-160 a_{4}\left(\dot{a}_{5}\right)^{3}+114\left(\dot{a}_{5}\right)^{4}\right) ;
\end{aligned}
$$

(ii) further, with the same constants $c_{i}{ }^{\prime}$, the coefficients of the first system must satisfy 4 nonlinear algebraic equations

$$
\left[\begin{array}{llll}
F_{44} & F_{43} & F_{42} & F_{41} \\
F_{34} & F_{33} & F_{32} & F_{31} \\
F_{24} & F_{23} & F_{22} & F_{21} \\
F_{14} & F_{13} & F_{12} & F_{11}
\end{array}\right]\left[\begin{array}{l}
c_{4} \\
c_{3} \\
c_{2} \\
c_{1}
\end{array}\right]=\left[\begin{array}{l}
0 \\
0 \\
0 \\
0
\end{array}\right],
$$

where

$$
\begin{aligned}
F_{i, j}= & \sum_{k=\max (1, i-j)}^{n} \sum_{s=\max (1, k-i+1)}^{\min (k, k-i+j+1)} \frac{k !}{s !(k-s) !} a_{k} f_{i+s-k-1, j}^{(s)} \\
& -\sum_{k=1}^{j} \sum_{s=\max (1, k-i+1)}^{k} \frac{k !}{s !(k-s) !} f_{k, j} a_{i+s-k-1}^{(s)} \text { for } i, j=4,3,2,1,0 .
\end{aligned}
$$

Proof. When the general conditions of Theorem 2.1 are applied for the commutativity of fifthorder systems, (5.1a) results simply as the matrix form of (2.4a) for $n=5$. The explicit solutions given in (5.1b) are obtained by evaluating the integrals in (2.4b) with $n=5$, for each $f_{i j}$ successively in the order given in (5.1b). However, it is very exhaustive to derive the explicit expressions for $f_{i j}$ given in this equation. The derivation details are wearying and too lengthy to include in the proof, though they involve an attentive treatment of the ordinary integration methods. But the verification is straight foreword by inserting them in $(2.4 b)$ for $n=5$. Finally (5.2a), (5.2b) follows as the direct result of the second condition (2.5a), (2.5b) of Theorem 2.1 for $n=5$ and it is included for the sake of completeness of the theorem.

The above equations in (5.1b) explicitly represent the coefficients $b_{i}$ 's of the same or lower order commutative systems in terms of the coefficients $a_{i}{ }^{\prime}$ s of a fifth-order system. Using these equations in (3.8a)-(3.8d) and inserting the results in (3.2a), (3.2b), (3.7a), $(3.7 b),(3.9 a),(3.9 b)$, the commutativity conditions for systems with initial conditions are also expressed explicitly. Although it appears that these explicit results are inevitably lengthy, (5.1a), (5.1b) and all the subsequent equations obtained from them greatly simplify if some of the coefficients $a_{i}$ 's are constant or completely zero. 
Another remark about shortening all the apparent results (not only for fifth-order systems) obtained in this paper is the assumption $a_{n}(t)=1$; that is, the normalization of the coefficients $a_{i}$ 's dividing by $a_{n}(t)$. In this case, all the derivatives of $a_{n}(t)$ will be zero, and all its integer or fractional powers will be 1 . But this is experienced complicating the expressions for the derivatives of the remaining coefficients due to the existence of the denominator term generated by normalization.

\section{Effects of Commutativity on System Sensitivity and Disturbance}

The importance of commutativity for system sensitivity was first reported by the author $[9,11]$. It was shown that when the order of connections of a second-order system and its first-order commutative pair was changed, the impulse response and hence the input-output transfer characteristics could be much more stable to parameter changes in one case than the other. This fact is very important in systems which use cascade connection of subsystems, which is usually the case in most of the telecommunication, control, and other practical applications. The effect of noise or disturbance interfering at an intermediate stage of a system may also depend on the order of connections of the subsystems. Therefore, the convenient order must be preferred when cascading the systems which need to be commutative to function the same input-output relation.

To illustrate the use of the derived formulas for the commutativity of fifth-order systems, the first example is chosen accordingly for the study of sensitivity and disturbance in conjunction with the commutativity.

Example 6.1. Let A be a fifth-order system described by

$$
\frac{d^{5}}{d t^{5}} y_{1}+t \frac{d^{4}}{d t^{4}} y_{1}+y_{1}=x_{1}
$$

Consider the search for the fifth- or lower-order commutative pairs of A. Computing $f_{i, j}$ 's expressed in (5.1b) and inserting them in (5.1a), the following matrix equation is obtained for the fifth- or lower-order commutative systems B:

$$
\left[\begin{array}{l}
b_{5} \\
b_{4} \\
b_{3} \\
b_{2} \\
b_{1} \\
b_{0}
\end{array}\right]=\left[\begin{array}{cccccc}
1 & 0 & 0 & 0 & 0 & 0 \\
t & 1 & 0 & 0 & 0 & 0 \\
0 & \frac{4 t}{5} & 1 & 0 & 0 & 0 \\
0 & \frac{-\left(10+2 t^{2}\right)}{25} & \frac{3 t}{5} & 1 & 0 & 0 \\
0 & \frac{\left(20 t+4 t^{3}\right)}{125} & \frac{-\left(15+3 t^{2}\right)}{25} & \frac{2 t}{2 t} & 1 & 0 \\
1 & \frac{\left(175-90 t^{2}-11 t^{4}\right)}{625} & \frac{\left(7 t^{3}+9 t\right)}{125} & \frac{-\left(15+3 t^{2}\right)}{25} & \frac{t}{5} & 1
\end{array}\right]\left[\begin{array}{l}
c_{5} \\
c_{4} \\
c_{3} \\
c_{2} \\
c_{1} \\
c_{0}
\end{array}\right] .
$$


In view of $(5.2 \mathrm{a}),(5.2 \mathrm{~b})$, the second commutativity condition of Theorem 2.1 implies

$$
\left[\begin{array}{cccc}
F_{44} & F_{43} & F_{42} & \frac{4 t}{5} \\
F_{34} & F_{33} & \frac{-6 t}{25} & 0 \\
F_{24} & \frac{42 t}{125} & 0 & 0 \\
\frac{-264 t}{125} & 0 & 0 & 0
\end{array}\right]\left[\begin{array}{l}
c_{4} \\
c_{3} \\
c_{2} \\
c_{1}
\end{array}\right]=\left[\begin{array}{l}
0 \\
0 \\
0 \\
0
\end{array}\right]
$$

where $F_{i, j}$ 's are computed using $(2.5 \mathrm{~b})$. Note that the entries above the second cross diagonal are not computed since they are not needed to arrive the conclusion that $c_{4}=c_{3}=c_{2}=c_{1}=0$. With this information, all the commutative pairs of $\mathrm{A}$ are described by

$$
c_{5}\left[\frac{d^{5}}{d t^{5}} y_{2}+t \frac{d^{4}}{d t^{4}} y_{2}+y_{2}\right]+c_{0} y_{2}=x_{2}
$$

Since $c_{n-1}=c_{n-2}=\cdots=c_{1}=0$, this case is obviously a special application of Corollary 3 in [1]. In fact, system B described by (6.4) has a block-diagram representation shown in Figure 3; that is, B is obtained from A by an arbitrary forward and feedback path gains $1 / c_{5}$ and $c_{0}$, respectively.

Consider now the commutativity with nonzero initial conditions. For the system $\mathrm{A}$ in (6.1) and the system B in (6.4), $m=n=5$ and Corollary 3.3 necessitates A and B have the same initial conditions in the null space of

$$
c_{5}\left(c_{0}+c_{5}-1\right)\left[\begin{array}{ccccc}
1 & 0 & 0 & 0 & 0 \\
-t & 1 & 0 & 0 & 0 \\
t^{2}-2 & -t & 1 & 0 & 0 \\
-t^{3}+3 t & t^{2}-1 & -t & 1 & 0 \\
t^{4}-4 t^{2}+2 & -t^{3}+2 t & t^{2}-1 & -t & 1
\end{array}\right]
$$

which is the coefficient matrix in (3.9b).

Obviously, the null space is empty for all initial times unless $c_{0}+c_{5}=1$, and for $c_{0}+c_{5}=$ $1, \mathrm{~A}$ and $\mathrm{B}$ are commutative for any set of initial conditions.

The above conclusions are verified by Simulink. The results are plotted in Figure 4. In this figure, the curve I shows the equal responses of $\mathrm{AB}$ and $\mathrm{BA}$ to a unit step for $c_{0}=3$, $c_{5}=0.2$ with zero initial conditions. For curves II-AB and BA, equal nonzero initial conditions $\left(y_{i}(0)=-1, \dot{y}_{i}(0)=2, i=1,2\right.$, all others are zero) are used with the same values of input and $c_{0}, c_{5}$; obviously A and B are not commutative since $c_{0}+c_{5} \neq 1$. In Figure 4(b), I shows the step response of both $\mathrm{AB}$ and $\mathrm{BA}$ with the equal initial conditions when $c_{0}=0.75, c_{5}=0.25$, that is, $c_{0}+c_{5}=1$; hence the commutativity conditions hold. When the same coefficients but unequal initial conditions are used $\left(\dot{y}_{1}(0)\right.$ is changed to 1$), \mathrm{AB}$ and $\mathrm{BA}$ yield different responses as shown by the curves II-AB, BA.

To show the sensitivities of $\mathrm{AB}$ and $\mathrm{BA}$ to parameter values and initial conditions, consider the commutative case $y_{i}(0)=-1, \dot{y}_{i}(0)=0.2, i=1,2, c_{0}=0.75, c_{5}=0.25$ for which 


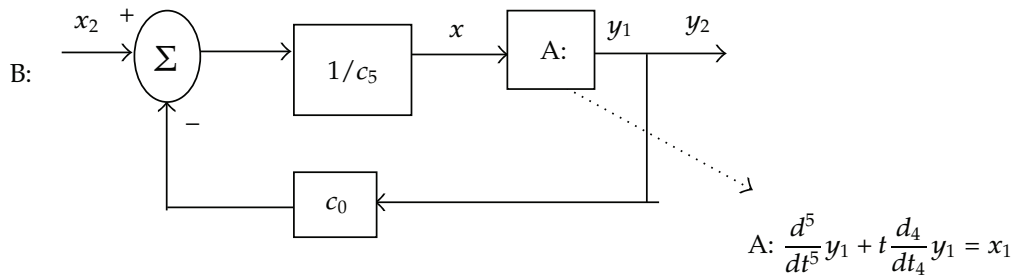

Figure 3: The only commutative class of systems of A considered in Example 6.1.

the unit step response was shown in Figure 4(b) (I). If the commutativity condition is spoiled slightly by changing $y_{1}(0)$ to -1.2 , then the responses of $A B$ and $B A$ change as shown in Figure $4(\mathrm{c})$ by the curves $\mathrm{I}-\mathrm{AB}, \mathrm{BA}$. Obviously, the connection $\mathrm{AB}$ is much more sensitive to $y_{1}(0)$ than $B A$. On the other hand, when $c_{0}$ is reduced to 0.60 , then $A B$ and $B A$ give the responses shown in Figure 4(c) by II-AB, BA; AB is still more sensitive than BA for this case.

To test the disturbance by an external noise, a unit step is injected in to the connection between $\mathrm{A}$ and $\mathrm{B}$ when both systems are initially relaxed and unforced with $c_{0}=0.75, c_{5}=$ 0.25 . Under the same conditions, a saw-tooth wave with period 0.2 and ranging from -18.5 to 20 is applied as the second disturbance. The results are shown in Figure 4(d) by the curves $\mathrm{I}-\mathrm{AB}, \mathrm{BA}$ and II-AB, BA, respectively. Comparing the results with the zero response, it is seen that the output of $A B$ is much more effected than that of $B A$ by both of the disturbances.

Example 6.2. Consider the second-order time-invariant linear systems

$$
\begin{aligned}
& \text { A: } \quad \ddot{y}_{1}-y_{1}=x_{1}, \\
& \text { B: } 2 \ddot{y}_{2}+b_{1} \dot{y}_{2}+b_{0} y_{2}=x_{2},
\end{aligned}
$$

where A is chosen unstable to emphasize on the effects of commutativity on sensitivity and disturbance. For the commutativity of these systems with nonzero initial conditions as well, Corollary 3.4 requires

$$
\begin{gathered}
Y_{A}=\left[\begin{array}{l}
y_{1}(0) \\
\dot{y}_{1}(0)
\end{array}\right]=Y_{B}=\left[\begin{array}{l}
y_{2}(0) \\
\dot{y}_{2}(0)
\end{array}\right] \\
{\left[\begin{array}{cc}
1.5+0.5 b_{0} & 0.5 b_{1} \\
0.25 b_{1}\left(1-b_{0}\right) & 1.5+0.5 b_{0}-0.25 b_{1}^{2}
\end{array}\right]\left[\begin{array}{l}
y_{1}(0) \\
\dot{y}_{1}(0)
\end{array}\right]=\left[\begin{array}{l}
0 \\
0
\end{array}\right] .}
\end{gathered}
$$

The coefficient matrix in (6.8) is the one given in (3.10) and obtained from it by using formulas in (4.2) for the given systems in this example. To have commutativity with nonzero initial conditions, it is necessary that this coefficient matrix must be singular which is achieved if

$$
b_{1}=\mp \sqrt{2}\left(1.5+0.5 b_{0}\right) \text {. }
$$




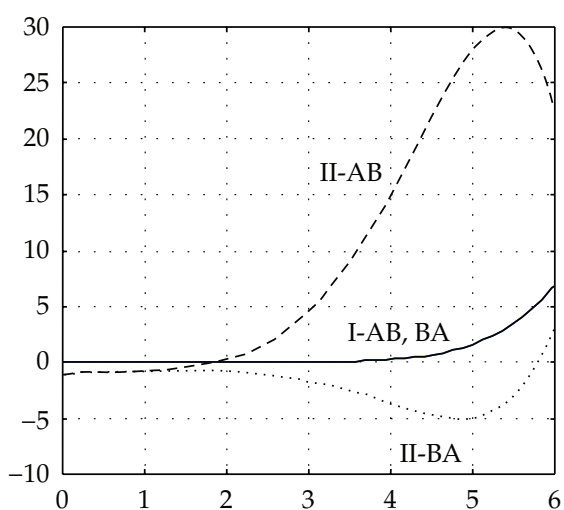

(a)

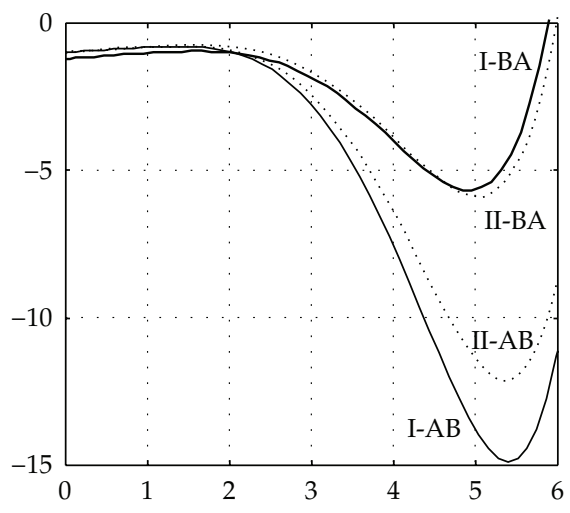

(c)

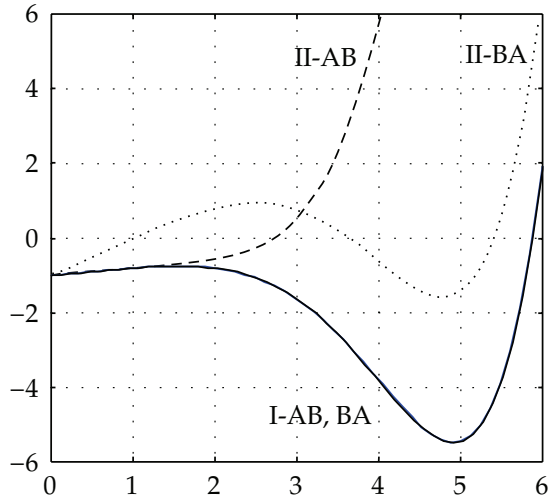

(b)

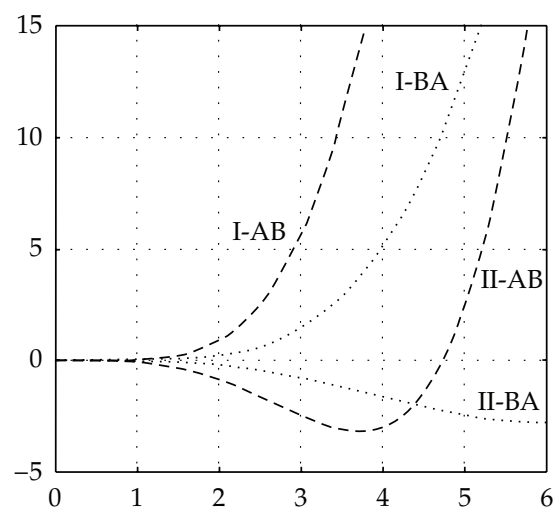

(d)

Figure 4: Simulation results for Example 6.1.

Further, (6.8) requires

$$
\left(1.5+0.5 b_{0}\right) y_{1}(0)+0.5 b_{1} \dot{y}_{1}(0)=0
$$

Obviously, if $b_{0}=3$, then $b_{1}=0$ and the systems are commutative for any set of equal initial conditions; that is, $y_{1}(0)=y_{2}(0)$ and $\dot{y}_{1}(0)=\dot{y}_{2}(0)$ can be chosen arbitrarily. If $b_{0} \neq-3$, using (6.9), (6.10) is reduced to

$$
\dot{y}_{1}(0)= \pm \sqrt{2} y_{1}(0) \text {. }
$$

It is important to note that all of these commutativity results can be equally obtained by taking the Laplace transform of (6.6) with the general nonzero initial conditions for both systems A and B; and then computing and equating the $s$-domain input-output relations for the connections $\mathrm{AB}$ and $\mathrm{BA}$. The result is

$$
Y_{\mathrm{AB}}=\frac{X+\left(2 y_{1}\right) s^{3}+\left(b_{1} y_{1}+2 \dot{y}_{1}\right) s^{2}+\left(2 y_{2}+b_{0} y_{1}+b_{1} \dot{y}_{1}\right) s+\left(b_{1} y_{2}+b_{0} \dot{y}_{1}+2 \dot{y}_{2}\right)}{(s-1)(s+1)\left(2 s^{2}+b_{1} s+b_{0}\right)}
$$




$$
Y_{\mathrm{BA}}=\frac{X+\left(2 y_{2}\right) s^{3}+\left(b_{1} y_{2}+2 \dot{y}_{2}\right) s^{2}+\left(y_{1}-2 y_{2}\right) s+\left(\dot{y}_{1}-2 \dot{y}_{2}-b_{1} y_{2}\right)}{(s-1)(s+1)\left(2 s^{2}+b_{1} s+b_{0}\right)},
$$

where $y_{1}, y_{2}, \dot{y}_{1}, \dot{y}_{2}$ represent the initial values at time $t_{0}=0$. Equating the appropriate coefficients of $Y_{\mathrm{AB}}$ and $Y_{\mathrm{BA}}$, results equivalent to the commutativity conditions in (6.7), (6.8) are obtained. However, this elementary process is not valid for time varying systems for which Laplace transform can apply only under specific conditions, and it is not as systematic and easily applicable as given in this paper for higher-order time-invariant systems.

The simulation results for the case $b_{0}=-3, b_{1}=0$ with a unit step input are shown in Figure $5(\mathrm{a})$. For $y_{i}(0)=1$ and $\dot{y}_{i}(0)=-1, i=1,2$, both connections yield the response I which is coincident with the plot of the analytic result

$$
y(t)=\frac{1}{3}\left[1-1.5 e^{t}-4.5 e^{-t}+(4-2 \sqrt{1.5}) e^{\sqrt{1.5} t}+(4+2 \sqrt{1.5}) e^{-\sqrt{1.5} t}\right]
$$

computed from (6.12) or (6.13) by setting $X=1 / s$ and inserting the given numerical values in; for $y_{i}(0)=-1$ and $\dot{y}_{i}(0)=1$, the responses for $\mathrm{AB}$ and $\mathrm{BA}$ are still equal as noted by II; when $b_{1}$ is changed to -2.5 in case II, the commutativity condition in (6.9) is spoiled and different responses are obtained as indicated by III-AB and III-BA for AB and BA. Further results are shown in Figure 5 for the case $b_{0} \neq-3$; with $b_{0}=9$, (6.9) implies $b_{1}=\mp 6 \sqrt{2}$; and with $b_{1}=6 \sqrt{2},(6.11)$ implies $\dot{y}_{1}(0)=-\sqrt{2} y_{1}(0)$. For $y_{i}(0)=1$ and $\dot{y}_{i}(0)=-\sqrt{2}, i=1,2$, it can be shown by using (6.12) or (6.13) that an input

$$
x(t)=2(\sqrt{2}-1) \sin t
$$

cancels the pole in (6.12), (6.13) at $s=1$, and hence it prevents unboundedness and produces an output

$$
y(t)=\frac{11+6 \sqrt{2}}{49} e^{-t}+\left[\frac{4110-432 \sqrt{2}}{5929}+\frac{15 \sqrt{2}-4}{77} t\right] e^{-1.5 \sqrt{2} t}+\frac{\sqrt{2}-1}{121}(12 \cos t-7 \sin t) .
$$

This analytical solution is shown in Figure 5(b) by I; in the same figure, II indicates the equal outputs of both $\mathrm{AB}$ and $\mathrm{BA}$ obtained by Simulink. A slight difference originates from the deficiency of Simulink due to the particular case in concern, that is, exact pole cancellation cannot be achieved numerically, so that simulation results get unbounded as $t \rightarrow \infty$. To test the sensitivity to the initial conditions, $y_{i}(0)$ is changed to 1.01 ; the result is that the output of $\mathrm{BA}$ blows up almost two times before that of $\mathrm{AB}$, as seen from the curves III-AB and III-BA in Figure 5(b), respectively; hence BA is more sensitive then $A B$ to $y_{i}(0)$. In Figure 5(c), the responses to $10 \sin (10 t)$ are compared for $\mathrm{AB}$ and $\mathrm{BA}$; I is obtained for $\mathrm{AB}$ and $\mathrm{BA}$ with the same commutativity conditions valid for Figure 5(b) (I and II); II-AB and II-BA are obtained for $\mathrm{AB}$ and $\mathrm{BA}$ when $b_{0}$ is increased from $6 \sqrt{2}$ to 10 . Obviously, $\mathrm{AB}$ is almost insensitive to this change and $B A$ is still more sensitive then $A B$. To show the disturbance effects, under the zero input conditions of the case in Figure 5(b) (I, II), a step of magnitude of 0.1 is injected at time $t=1$ between $\mathrm{A}$ and $\mathrm{B}$. The output without disturbance (I-AB, BA) and the output 


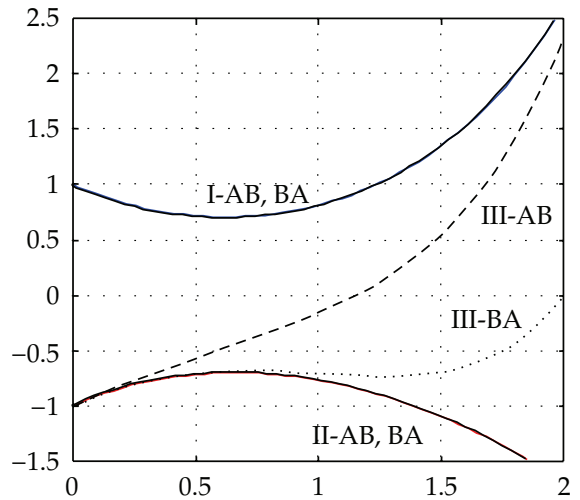

(a)

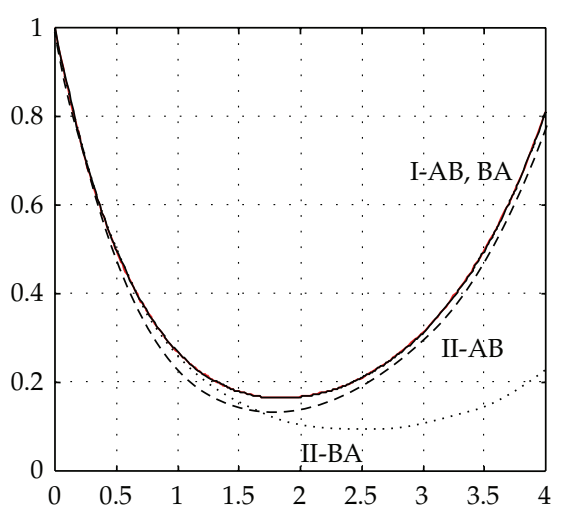

(c)

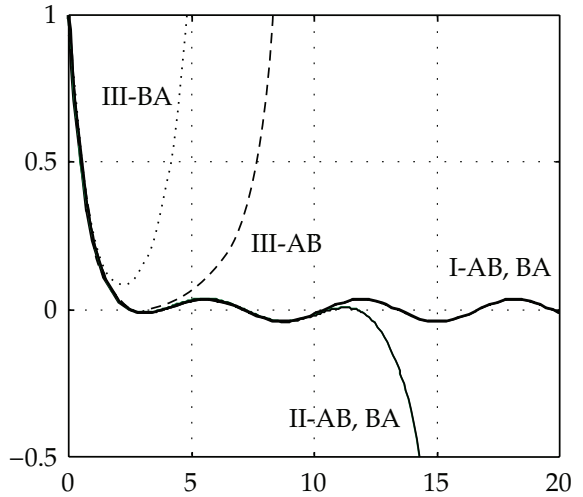

(b)

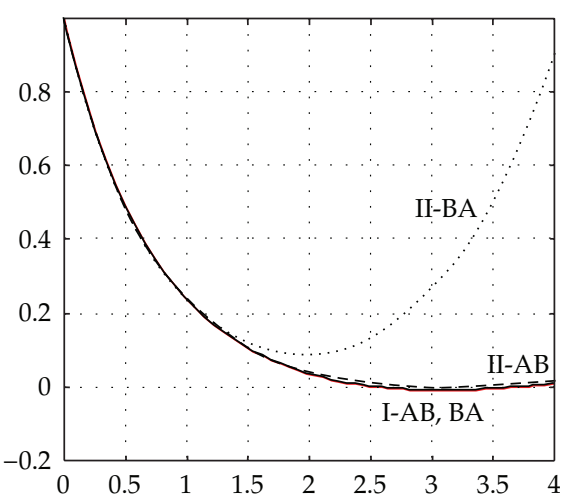

(d)

Figure 5: Simulation and analytical results for Example 6.2.

with disturbance (II-AB, II-BA) are shown in Figure 5(d); obviously, AB is almost insensitive to this disturbance and BA is much more affected then $A B$.

Example 6.3. This example is preciously chosen so that the systems are both time varying and they have analytic solutions. A is defined as

$$
\text { A: } \quad \ddot{y}_{1}+2 t \dot{y}_{1}+t^{2} y_{1}=x_{1}
$$

The coefficients of this system satisfy the first set of commutativity conditions expressed by (2.5a), and with (2.4a) all of its commutative pairs are expressed by

$$
\text { B: } \quad c_{2} \ddot{y}_{2}+\left(2 c_{2} t+c_{1}\right) \dot{y}_{2}+\left(c_{2} t^{2}+c_{1} t+c_{0}\right) y_{2}=x_{2} \text {. }
$$




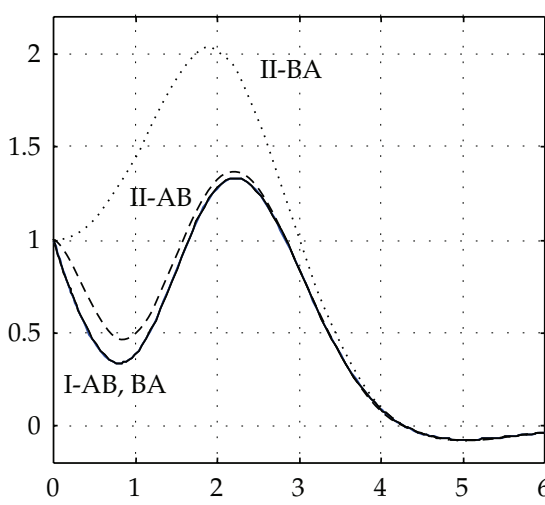

(a)

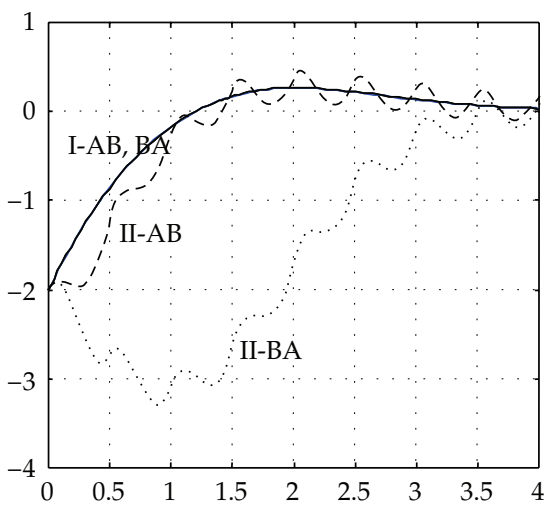

(c)

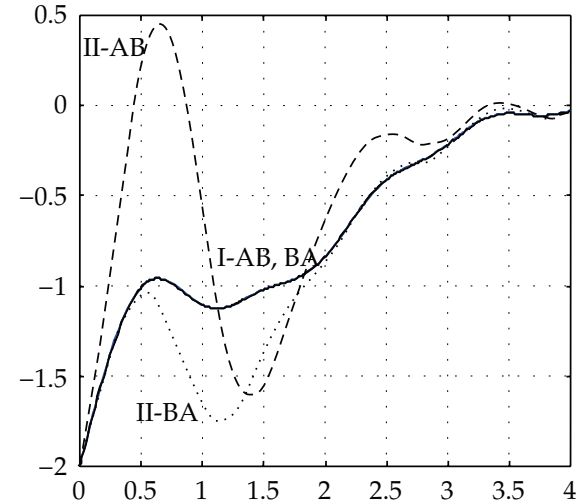

(b)

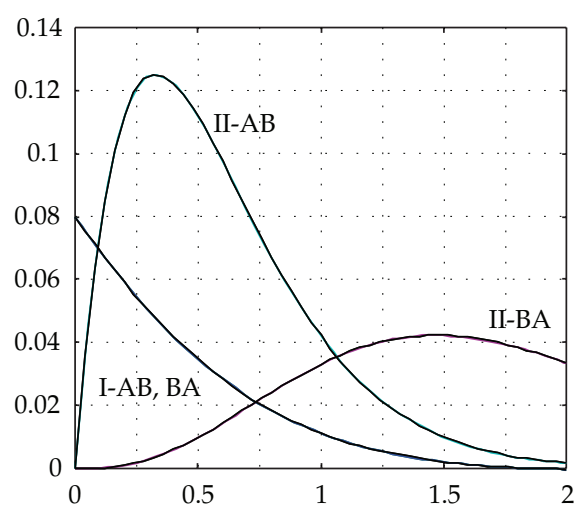

(d)

Figure 6: Simulation and analytical results for Example 6.3.

Further, if $\mathrm{A}$ and $\mathrm{B}$ are required to be commutative with nonzero initial conditions as well, Corollary 3.3 requires

$$
\left[\begin{array}{l}
y_{1} \\
\dot{y}_{1}
\end{array}\right]=\left[\begin{array}{l}
y_{2} \\
\dot{y}_{2}
\end{array}\right],
$$

$$
\frac{1}{c_{2}^{2}}\left[\begin{array}{cc}
c_{2} c_{1} t+c_{2}^{2}-c_{2}+c_{2} c_{0} & c_{2} c_{1} \\
-3 c_{2} c_{1} t^{2}-\left(2 c_{2}^{2}+c_{1}^{2}-2 c_{2}\right) t+c_{1}\left(1+c_{2}-c_{0}\right) & -3 c_{1} c_{2} t+c_{2}^{2}-c_{1}^{2}-c_{2}+c_{2} c_{0}
\end{array}\right]\left[\begin{array}{l}
y_{1} \\
\dot{y}_{1}
\end{array}\right]=0
$$

at the initial time $t=t_{0}$. For the commutativity with nonzero initial conditions, the coefficient matrix in (6.18b) must be singular, that is, its determinant must be zero and this is achieved if and only if

$$
c_{2}+c_{0}-1=\mp \sqrt{2} c_{1} .
$$


With this condition, $(6.18 \mathrm{~b})$ implies the relation

$$
\dot{y}_{1}\left(t_{0}\right)=-\left(t_{0} \mp \sqrt{2}\right) y_{1}\left(t_{0}\right)
$$

between equal initial conditions of systems A and B, so that they are commutative in case of nonzero initial conditions as well.

For the analytic solution of differential equations describing systems A and B, these equations are first written in state-space form, then the successive decomposition method is used to decompose the state-coefficient $A$-matrix $[13,14]$. A second decomposition is used to evaluate the state transition matrix $\phi(t, \tau)[15]$. Knowing the state transition matrices, the general solutions with arbitrary initial conditions at $t_{0}=0$ are obtained for systems $\mathrm{A}$ and $\mathrm{B}$ by the usual methods. The computed results are expressed for $c_{2}=1$ as

$$
\begin{gathered}
y_{1}(t)=e^{-0.5 t^{2}}\left[y_{1}(0) \cosh t+\dot{y}_{1}(0) \sinh t+\int_{0}^{t} e^{0.5 \tau^{2}} \sinh (t-\tau) x_{1}(\tau) d \tau\right] \\
y_{2}(t)=\frac{1}{b} e^{-0.5 t^{2}-0.5 c_{1} t}\left[y_{2}(0)\left(b \cosh b t+0.5 c_{1} \sinh b t\right)+\dot{y}_{2}(0) \sinh b t\right. \\
\left.+\int_{0}^{t} e^{0.5 \tau^{2}+0.5 c_{1} \tau} \sinh b(t-\tau) x_{2}(\tau) d \tau\right]
\end{gathered}
$$

where $b=+\sqrt{1-c_{0}+0.25 c_{1}^{2}}$. Obviously both systems are strongly stable due to the terms $-0.5 t^{2}$ in the exponents.

Using the input-output relations for the connections AB and BA depicted in Figure 1, the analytic solutions are obtained for any input $x(t)$ and any set of initial conditions. The derivation details and the results are not given here for space limitations. Comparison of the like terms in the solutions reveals that the responses with zero initial conditions are equal. Further, to have equivalence with nonzero initial conditions, it is verified that (6.19a), (6.19b) must hold, and A and B must have identical initial conditions satisfying (6.19b).

The simulation results and some analytical solutions for the example are shown in Figure 6. In this figure, equal initial conditions are used for A and B in all cases; it is set that $c_{2}=1, c_{1}=4 \sqrt{2}, c_{0}=8$ as to satisfy the commutativity conditions. In Figure $6(\mathrm{a})$, the input is $100 \sin t$; I is for $y_{1}(0)=1, \dot{y}_{1}(0)=-\sqrt{2}$ so that $(6.19 \mathrm{~b})$ is satisfied; hence $\mathrm{AB}$ and BA give the same response. However, when $\dot{y}_{1}(0)$ is set to 0 , then $(6.19 \mathrm{~b})$ is spoiled and AB and BA give different responses as shown by the curves II-AB and II-BA. Observe that the connection $\mathrm{BA}$ is more sensitive to this change than $\mathrm{AB}$. In Figure $6(\mathrm{~b})$, the input is a square wave of magnitude 100 and period $1, y_{1}(0)=-2, \dot{y}_{1}(0)=2 \sqrt{2}$; since all the commutativity conditions hold $\mathrm{AB}$, and $\mathrm{BA}$ yield the same responses $\mathrm{I}-\mathrm{AB}, \mathrm{BA}$; however, when the coefficient $c_{1}$ in $(6.17 \mathrm{~b})$ is omitted, the first commutativity condition gets violated and the connections give different responses indicated by II-AB and II-BA. To see the disturbance (or noise) effects, the input is set to $10, y_{1}(0)=-2, \dot{y}_{1}(0)=2 \sqrt{2}$. Commutativity conditions hold and both connections yield the response shown in Figure 6(c) by the curve I-AB, BA. But when a saw tooth disturbance signal (with period 0.5 , maximum and minimum values \pm 50 ) is injected between the connections, $\mathrm{BA}$ is much more affected than $\mathrm{AB}$ as the responses II-AB and IIBA show. Finally in Figure 6(d), the curve I shows the output (scale 100:1) of AB and BA 
with zero input and equal initial conditions $y_{1}(0)=y_{2}(0)=8, \dot{y}_{1}(0)=\dot{y}_{2}(0)=-8 \sqrt{2}$; the results for $\mathrm{AB}, \mathrm{BA}$, and the analytic solution

$$
y(t)=e^{-0.5 t^{2}}\left[4 \cosh t-3 \sqrt{2} \sinh t+e^{-2 \sqrt{2} t}(4 \cosh t+3 \sqrt{2} \sinh t)\right]
$$

are all coincident. When $\dot{y}_{2}(0)$ is increased by an amount of $\varepsilon$, the deviations $\Delta y_{\mathrm{AB}}$ and $\Delta y_{\mathrm{BA}}$ analytically expressed by

$$
\begin{gathered}
\Delta y_{\mathrm{AB}}=\varepsilon e^{-0.5 t^{2}-2 \sqrt{2} t} \sinh t \\
\Delta y_{\mathrm{BA}}=\frac{\varepsilon}{8} e^{-0.5 t^{2}}\left[-\sqrt{2} \cosh t+2 \sinh t+e^{-2 \sqrt{2} t}(\sqrt{2} \cosh t-2 \sinh t)\right]
\end{gathered}
$$

and the simulation results are plotted for $\varepsilon=1$ (curves II-AB, II-BA). Analytical and simulation results are checked to be coincident. Obviously, the connection BA is more sensitive than $\mathrm{AB}$ to the initial condition relevant to the derivative.

\section{Conclusions}

Commutativity conditions of two linear time-varying systems with nonzero initial conditions are thoroughly investigated in this paper. The derived conditions can be used to test the commutativity without solving the differential equations describing the system. Although equivalent conditions can be obtained in another way comparing the analytic solutions of the interconnections, very few classes of time-varying systems have explicit analytic solutions only. Therefore, the method of finding explicit solutions is not general and easily applicable as the method presented in this paper since it does not require finding the analytic solutions. This method can be used for all linear time varying systems as well.

The explicit commutativity conditions for fifth-order systems are obtained by an enormous effort and presented in this paper. Moreover, the commutativity is studied in view of system sensitivity and disturbance; it is shown that for commutative pairs, one order of connection might be more advantages than the other, which is an important fact from the applications point of view.

Most of the results are verified by examples treated analytically and/or by Simulink. It is seen that the analytical solutions and the results of Simulink are almost same with an exception where there is a perfect pole cancellation which cannot be achieved numerically by Simulink.

\section{References}

[1] M. Koksal, "An exhaustive study on the commutativity of time varying systems," International Journal of Control, vol. 47, no. 5, pp. 1521-1537, 1988.

[2] E. Marshall, "Commutativity of time varying systems," Electronics Letters, vol. 13, no. 18, pp. 539-540, 1977.

[3] M. Koksal, "Commutativity of second order time varying systems," International Journal of Control, vol. 36, no. 3, pp. 541-544, 1982. 
[4] S. V. Saleh, "Comments on 'Commutativity of second-order time-varying systems'," International Journal of Control, vol. 37, pp. 1195-1 196, 1983.

[5] M. Koksal, "Corrections on 'commutativity of second order time varying systems'," International Journal of Control, vol. 38, no. 1, pp. 273-274, 1983.

[6] M. Koksal, "General conditions for commutativity of time-varying systems," in Proceedings of the (IASTED) International Association of Science and Technology for Development Conference, Telecommunication and Control (TELECON '84), pp. 225-229, Halkidiki, Greece, 1984.

[7] M Koksal, A Survey on the Commutativity of Time-Varying Systems, Middle East Technical University, Gaziantep, Turkey, 1985.

[8] M. Koksal, "Commutativity of 4th order systems and Euler systems," in Proceedings of the National Congress of Electrical Engineering, Adana, Turkey, 1985, Paper no:BI-6.

[9] M. Koksal, "Effects of initial conditions on the commutativity and effects of commutativity on the system sensitivity," in Proceedings of the 2nd National Electrical Engineering Conference, pp. 570-573, Ankara, Turkey, 1987.

[10] M. Koksal, "Effects of nonzero initial conditions on the commutativity of linear time-varying systems," in Proceedings of the International Conference on Modelling and Simulation (AMSE '88), vol. 1, pp. 49-55, AMSE Press, Istanbul, Turkey, 1988.

[11] M. Koksal, "Effects of commutativity on system sensitivity," in Proceedings of the 6th International Symposium on Networks, Systems and Signal Processing (ISYNT2 '89), pp. 61-62, Zagreb, Yogoslavia, 1989.

[12] C. A. Desoer, Notes for a Second Course on Linear Systems, Van Nostrand Rheinhold, New York, NY, USA, 1970.

[13] M. Y. Wu, "A successive decomposition method for the solution of linear time-varying systems," International Journal of Control, vol. 33, no. 1, pp. 181-186, 1981.

[14] M. Y. Wu, "On the commutative class of linear time-varying systems," International Journal of Control, vol. 23, no. 3, pp. 433-444, 1976.

[15] M. Y. Wu and M. Horowitz, "On solution, stability and transformation of linear time-varying systems," International Journal of Control, vol. 22, no. 2, pp. 169-180, 1975. 


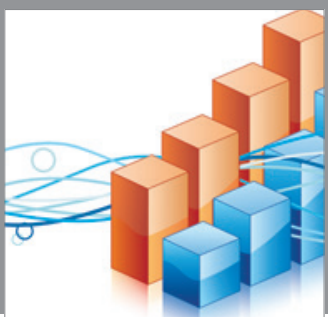

Advances in

Operations Research

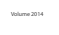

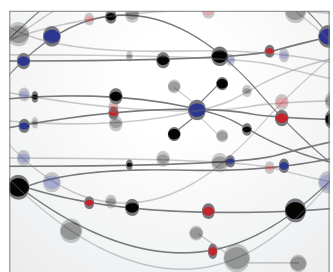

\section{The Scientific} World Journal
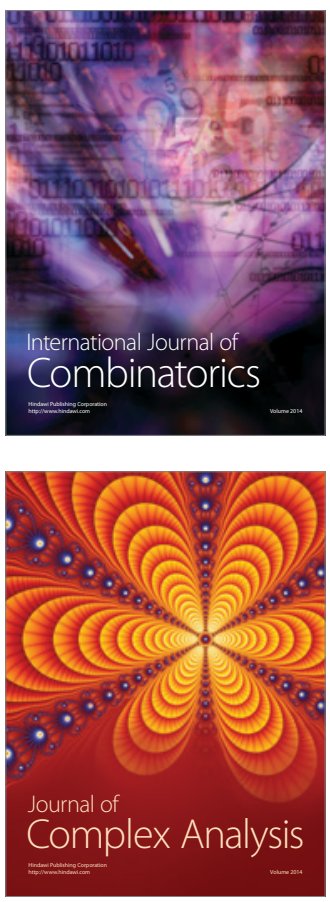

International Journal of

Mathematics and

Mathematical

Sciences
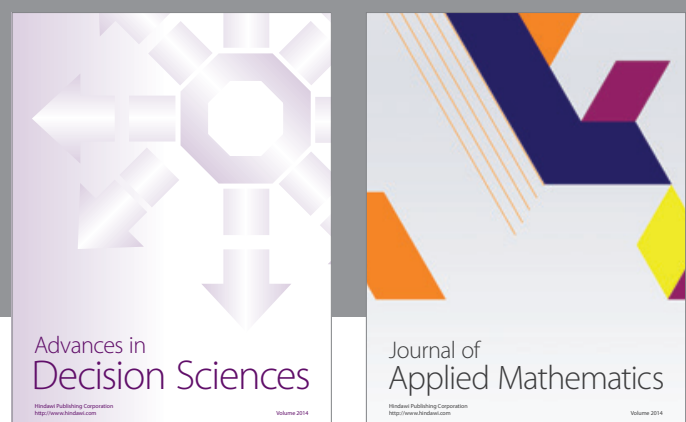

Journal of

Applied Mathematics
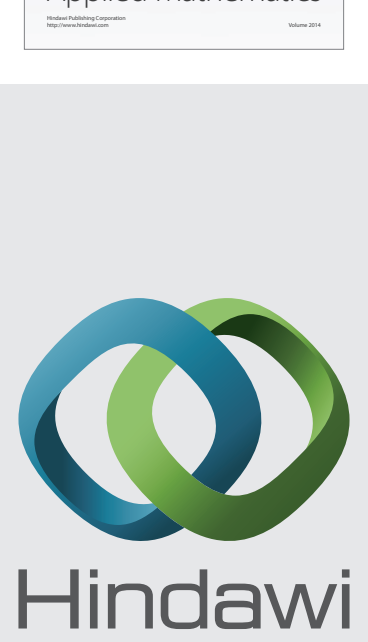

Submit your manuscripts at http://www.hindawi.com
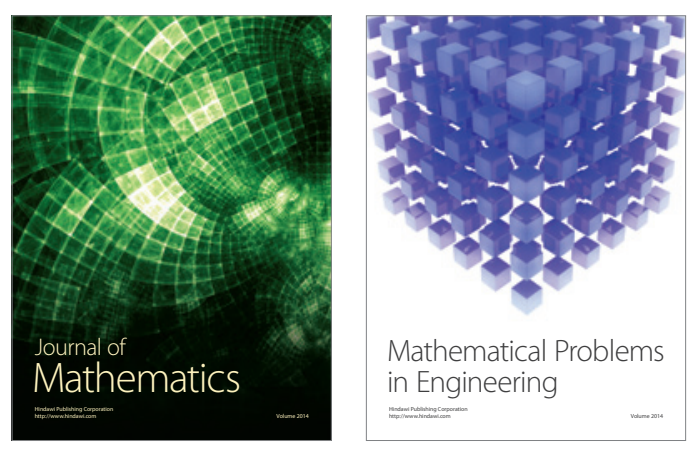

Mathematical Problems in Engineering
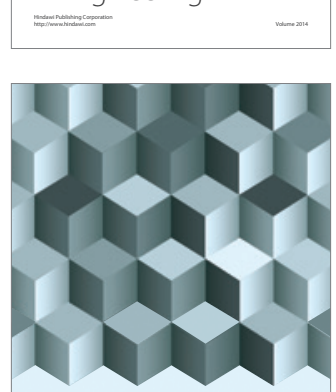

Journal of

Function Spaces
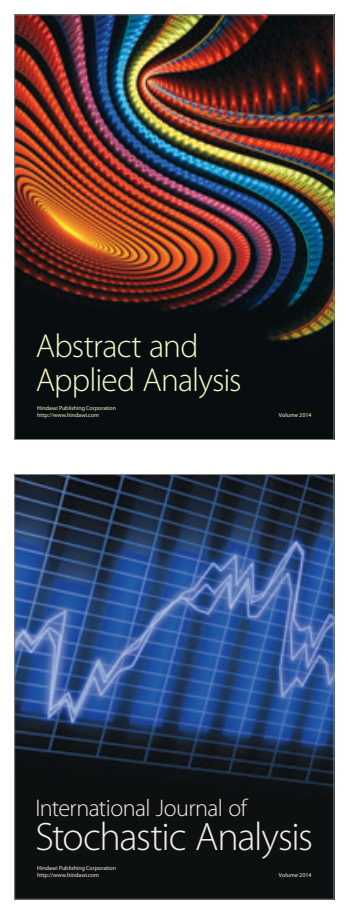

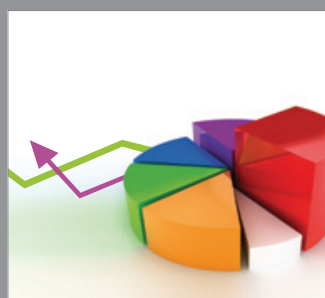

ournal of

Probability and Statistics

Promensencen
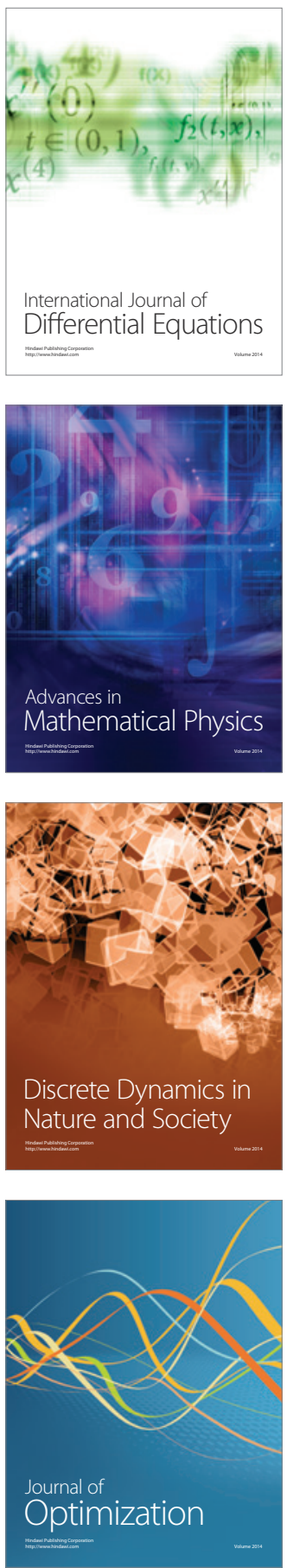